\title{
AVALIAÇÃO DA QUALIDADE DE VIDA EM PORTADORES DE PTERÍGIO OCULAR NO MUNICÍPIO DE PALMAS
}

\author{
EVALUATION OF QUALITY OF LIFE IN PATIENTS WITH OCULAR \\ PTERYGIUM IN THE MUNICIPALITY OF PALMAS
}

\section{Marco Túlio Chater Viegas ${ }^{a}$, Sandra Maria Botelho Pinheirob ${ }^{b}$ Fabiana Richa Valim Viegas ${ }^{a}$, Adojhones Frankcian da Silva Santos ${ }^{c}$, Wande Gonçalves Diniz ${ }^{\text {, }}$ Núbia Cristina de Freitas Maia ${ }^{\mathrm{e}}$}

amt.viegas@bol.com.br, 'bsandrabotelho@uft.edu.br, cadojhones.santos@gmail.com, dwande.diniz@live.com, enubiamaia@uol.com.br Universidade Federal do Tocantins - Palmas (TO), Brasil

Data de recebimento do artigo: 02/12/2014

Data de aceite do artigo: 17/08/2015

\section{RESUMO}

Introduçáo: $\mathrm{O}$ pterígio é caracterizado por um crescimento de tecido fibrovascular da conjuntiva bulbar sobre a córnea. É uma doença com maior frequência em indivíduos de países de clima tropical, acometendo principalmente homens com idade superior a 30 anos. Objetivo: Avaliar a qualidade de vida de portadores de diferentes tamanhos de pterígio no município de Palmas (TO). Materiais e métodos: Estudo observacional, transversal com 60 pacientes, alocados em 3 grupos de 20 pacientes cada. Os grupos foram organizados de acordo com o grau do pterígio, ficando nos grupos I, II ou III - pacientes com pterígio grau 1, 2 e 3, respectivamente. Foi aplicado um questionário epidemiológico e um sobre qualidade de vida (NEI VFQ-25), e os dados foram submetidos à análise estatística. Resultados: Ao analisarmos a acuidade visual (AV) e o escore de qualidade de vida entre os grupos em função do grau do pterígio, encontramos que no grupo I a AV em LogMAR foi de 0,015 $( \pm 0,067)$; no grupo II, a AV foi de 0,080 $( \pm 0,132)$; e no grupo III foi de $0,067( \pm 0,127)$, com $p=0,12$. Conclusóes: Houve alteração dos escores de qualidade de vida em ambos os grupos estudados, porém sem significância estatística entre eles, embora tenham sido encontradas diferenças na avaliação subjetiva da qualidade visual entre os grupos. Sugerimos novos estudos com maior número de participantes.

Palavras-chave: Pterígio; qualidade de vida; questionários; análise estatística; acuidade visual.

\section{ABSTRACT}

Introduction: Pterygium is characterized by growth of fibrovascular tissue of the bulbar conjunctiva on the cornea. It is a disease with greater frequency in individuals of tropical countries, affecting mainly men aged over 30 years. Objective: This study aimed to evaluate the quality of life of patients with different sizes of pterygium, in the city of Palmas/TO, Brazil. Materials and methods: Observational, cross-sectional study with 60 patients, allocated into 03 groups (I, II, and III) of 20 patients each, which were organized according to the degree of pterygium $(1,2$, and 3, respectively). An epidemiological questionnaire and another on quality of life (NEI VFQ-25) were completed, and the data was submitted to statistical analysis. Results: When comparing the visual acuity and quality of life scores among the groups, according to the degree of pterygium, it was observed that in Group I, LogMAR (Logarithm of the Minimum Angle of Resolution) visual acuity was $0.015( \pm 0.067)$; in group II, visual acuity was $0.080( \pm 0.132)$; and in group III was $0.067( \pm 0.127)$, with $\mathrm{p}=0.12$. Conclusion: There was a change in quality of life scores in both groups, but without statistical significance among them, although differences have been found in the subjective assessment of visual quality among groups. We recommend further studies with a larger number of participants.

Keywords: Pterygium; quality of life; health questionnaires; statistical analysis; visual acuity. 


\section{Introdução}

Pterígio é uma doença que afeta a superfície ocular dos portadores, caracterizada clinicamente por invasão centrípeta de tecido neovascular sobre a córnea, com diferentes graus de inflamaçáo associados ${ }^{1}$.

Embora a patogênese ainda seja incerta, existem fatores que podem estar associados, tais como clima seco e quente, poeira, vento, proximidade com a linha do Equador e alta luminosidade. Há evidências de que a exposição crônica à radiação ultravioleta e a infecção pelo papiloma vírus humano também estejam relacionadas na patogênese do pterígio ${ }^{2}$. Foi observada incidência de 4\% no Qatar ${ }^{3}, 5,2 \%$ na Índia ${ }^{4}$ e 6,2\% na Austrália ${ }^{5}$. Já a prevalência em comunidades ribeirinhas do Amazonas foi de 21,2\% ${ }^{6}$.

O crescimento do pterígio sobre a córnea, além de causar sintomas como sensação de corpo estranho, ardor, irritação e lacrimejamento, pode desencadear astigmatismo e consequente alteração visual. Isso acontece devido ao aplanamento focal e às modificaçóes na curvatura corneana, o que ocasiona déficit visual e reduz a produtividade laboral, social e funcional dos portadores, fator que pode alterar a qualidade de vida dos afetados ${ }^{7}$.

A qualidade de vida é um termo amplo, que inclui não somente aspectos relacionados à saúde geral (físicos, emocionais e mentais), mas também fatores não diretamente relacionados, como trabalho, família, ambiente social e outras circunstâncias da vida. Logo, qualidade de vida é mais do que uma descrição do estado de saúde do paciente, é uma reflexão de como ele percebe e reage ao seu estado de saúde e aos aspectos não médicos de sua vida ${ }^{8}$. Uma diversidade de instrumentos para medir a qualidade de vida tem sido desenvolvida. A maioria desses instrumentos era aplicável apenas à cultura para a qual foram desenvolvidos. Nos últimos anos, houve $\mathrm{o}$ interesse em desenvolver instrumentos que pudessem ser utilizados em diferentes culturas 9

A medida da qualidade de vida pode fornecer subsídios adicionais para auxiliar a decisão do momento exato de intervir com um procedimento terapêutico. Nesse contexto, entende-se a importância de relacionar assuntos de qualidade de vida em pesquisas de saúde ou mesmo em áreas específicas, como a oftalmologia ${ }^{10}$. É importante a aplicação de instrumentos já validados na língua local e que possam ser reprodutíveis na população estudada. A versão brasileira do Questionário de Funçáo Visual de 25 Itens (NEI VFQ-25) apresenta propriedades psicométricas válidas e confiáveis, e pode ser aplicada em pesquisa de qualidade de vida visual; portanto, tal questionário foi escolhido para a aplicação na população estudada ${ }^{11}$.
O objetivo desta pesquisa foi avaliar a qualidade de vida de portadores de pterígio quanto à acuidade visual de pacientes no município de Palmas (TO). Faz-se necessário o conhecimento da forma pelo qual essa doença afeta a vida das pessoas, seja dificultando a leitura ou o ato de dirigir, ou prejudicando a relaçáo interpessoal por motivos estéticos, uma vez que conhecer as implicações do próprio pterígio também é uma forma de prevenir e de tratar. É importante destacar que não existem trabalhos na literatura que fazem referência a esses aspectos, sendo inédito o estudo no estado do Tocantins.

\section{Metodologia}

Trata-se de um estudo observacional e transversal, realizado no ambulatório de oftalmologia do Centro de Referência Oftalmológica de Palmas (Cerop), no mês de agosto de 2013.

Após prévia autorização dos pacientes por meio da entrega do termo de consentimento livre e esclarecido, conforme preconiza a resolução 196/96 do Conselho Nacional de Saúde, iniciou-se a coleta dos dados e o exame oftalmológico. Para os analfabetos, o termo foi lido na presença de uma testemunha.

Os pacientes que consentiram participar da pesquisa foram submetidos a um exame oftalmológico e responderam a questionários aplicados pelo mesmo pesquisador, com um intervalo de sete dias entre a primeira e a segunda entrevista. $\mathrm{O}$ objetivo foi testar as respostas às questôes aplicadas. $\mathrm{Na}$ existência de diferenças significativas entre as respostas a uma mesma pergunta (diferenças acima de $50 \%$ na pontuação de cada questão), esta era então descartada e não serviu como pontuação para o cálculo do escore final.

O exame oftalmológico completo foi realizado pelo mesmo médico oftalmologista em 60 pacientes $(n=60)$. $\mathrm{O}$ exame incluiu a medida da acuidade visual com correção (utilizou-se a tabela de Snellen a uma distância de 6 metros), tonometria, biomicroscopia com descrição do tamanho do pterígio, fundoscopia, ceratometria e refração automatizada. Para a classificação do pterígio foi adotado como critério o grau de invasáo na córnea em milímetros, sendo grau I pterígios até $2 \mathrm{~mm}$ além do limbo, grau II pterígios entre 2 e $4 \mathrm{~mm}$ além do limbo e grau III aqueles pterígios que avançavam sobre a área pupilar $^{12}$.

Os pacientes foram subdivididos em três grupos com 20 pacientes alocados em cada um. O grupo 1 foi composto por portadores de pterígio grau I unilateral ou portadores de pterígio bilateral grau I. O grupo 2 foi composto por portadores de pterígio unilateral grau II ou bilateral, sendo que um dos olhos era no máximo classificado como grau II. O grupo 3 foi composto por 
portadores de pterígio grau III unilateral ou bilateral, sendo que um dos olhos era obrigatoriamente grau III.

Os critérios de inclusão foram pacientes com pterígio primário (sem intervençóes cirúrgicas) de qualquer tamanho, que aceitaram as condiçóes descritas no termo de consentimento da pesquisa.

Os critérios de exclusão foram pacientes portadores de outras doenças oculares associadas, tais como glaucoma, retinopatias, maculopatias, cicatrizes corneanas e pacientes com pterígios recidivados ou que faziam uso de colírios de prostaglandinas (pró-inflamatórios).

Após o exame oftalmológico foram aplicados dois questionários, um sobre dados epidemiológicos e outro sobre qualidade de vida. No questionário sobre dados epidemiológicos (questionário 1), foram descritos dados como sexo, raça, idade, local de residência, hábitos sociais de vida, tipo de atividade profissional e uso ou não de proteção solar, bem como o exame oftalmológico completo. $\mathrm{O}$ segundo questionário sobre qualidade de vida foi composto por três partes: saúde geral e visão, dificuldades com atividades e reaçóes aos problemas de visão.

Foram obtidos escores de acordo com as respostas obtidas por cada pergunta realizada, com variância de 0 a $100(0,25,50,75,100)$, de acordo com as especificaçóes de cálculo já padronizadas para o instrumento em questáo. A pontuação final obtida foi dividida pelo número de questóes respondidas. Nas questóes em que havia seis possibilidades de resposta, e caso o paciente escolhesse a última opção (número 6), optou-se por não computar esse dado no escore total, pois ele se refere à falta de interesse em determinada atividade por motivos não relacionados à visão. Por definição, quanto maior for o escore melhor é a qualidade de vida e a função visual do paciente.

Os dados coletados foram apresentados em tabelas de contingência e estratificados em três grupos de acordo com o grau de pterígio. Para a análise de dados de acuidade visual foi utilizada melhor visão (com correção) do olho com pterígio a fim de comparar os três grupos. Foi também realizada análise comparativa dos escores de qualidade de vida entre os casos de pterígio unilateral versus bilateral.

Após a coleta dos dados, os mesmos foram agrupados em tabelas por meio do programa Microsoft Excel'. Já para a realização da análise estatística posterior utilizou-se o software Stata versão11 (College Station, Texas, EUA).

Dados contínuos foram apresentados como média (desvio-padrão) e comparados entre os grupos por meio do teste de Kruskall-Wallis. Dados categóricos foram apresentados como frequência (proporção) e comparados com o teste exato de Fisher. Valores de p menores que 0,05 foram considerados estatisticamente significantes.

\section{Resultados}

A média de idade foi de $36,1( \pm 13,1)$ anos para o grupo I; 41( $\pm 9,2)$ anos para o grupo II e 48,2 $( \pm 15,8)$ anos para o grupo III, com diferença estatística comprovada entre os grupos I e III $(\mathrm{p}=0,04)$. Em relação ao sexo foram encontradas frequências de $35 \%, 45 \%$ e $45 \%$ para o sexo masculino e $65 \%$, $55 \%$ e $55 \%$ para o sexo feminino entre os grupos I, II e III, respectivamente. Quanto à raça, no grupo I, 8 (40\%) pacientes eram brancos, 8 (40\%) negros e 4 (20\%) pardos; no grupo II, $5(25 \%)$ brancos, $9(45 \%)$ negros e $6(30 \%)$ pardos; e no grupo III, $10(50 \%)$ brancos e $10(50 \%)$ negros. A maioria dos pacientes avaliados pertencia à zona urbana, sendo que 18 (90\%) do grupo I, 18 (94\%) do grupo II e $13(65 \%)$ do grupo III residiam na cidade (Tabela 1).

Tabela 1: Distribuição dos grupos analisados em relação a idade, sexo, raça e residência.

\begin{tabular}{|c|c|c|c|c|}
\hline $\begin{array}{l}\text { Idade } \\
\text { (anos) }\end{array}$ & $\begin{array}{c}\text { Grupo I } \\
36,1 \\
(13,1)\end{array}$ & $\begin{array}{l}\text { Grupo II } \\
41,0(9,2)\end{array}$ & $\begin{array}{c}\text { Grupo III } \\
48,2 \\
(15,8)\end{array}$ & $\begin{array}{c}\text { p } \\
0,04\end{array}$ \\
\hline \multicolumn{5}{|l|}{ Sexo } \\
\hline Masculino & $7(35 \%)$ & $9(45 \%)$ & $9(45 \%)$ & \multirow[b]{2}{*}{0,85} \\
\hline Feminino & $13(65 \%)$ & $11(55 \%)$ & $11(55 \%)$ & \\
\hline \multicolumn{5}{|l|}{ Raça } \\
\hline Branca & $8(40 \%)$ & $5(25 \%)$ & $10(50 \%)$ & \multirow{3}{*}{0,08} \\
\hline Negra & $8(40 \%)$ & $9(45 \%)$ & $10(50 \%)$ & \\
\hline Parda & $4(20 \%)$ & $6(30 \%)$ & $0(0 \%)$ & \\
\hline \multicolumn{5}{|l|}{ Residência } \\
\hline Urbana & $18(90 \%)$ & $18(94 \%)$ & $\begin{array}{c}13(65 \%) \\
7(35 \%)\end{array}$ & 0,06 \\
\hline
\end{tabular}

Testes estatísticos realizados através do teste exato de Fisher.

A maioria dos pacientes possuía segundo grau completo, sendo 9 (45\%) do grupo I, 12 (60\%) do grupo II e $14(70 \%)$ pacientes do grupo III. Foi observada alta frequência de não uso de proteção solar - sejam óculos ou chapéu - entre os três grupos, sendo 15 (75\%), 13 (65\%) e 14 (70\%) nos grupos I, II e III, respectivamente. A maioria dos pacientes não era tabagista ou etilista (Tabela 2). 
Tabela 2: Distribuição dos grupos analisados em relação ao grau de instrução, proteção solar e hábitos.

\begin{tabular}{|c|c|c|c|c|}
\hline & Grupo I & Grupo II & Grupo III & $\mathbf{p}$ \\
\hline \multicolumn{5}{|l|}{$\begin{array}{c}\text { Grau de } \\
\text { Instruçáo }\end{array}$} \\
\hline Analfabeto & $0(0 \%)$ & $1(5 \%)$ & $2(10 \%)$ & \multirow{4}{*}{0,32} \\
\hline $1^{\circ} \mathrm{Grau}$ & $6(30 \%)$ & $5(25 \%)$ & $3(15 \%)$ & \\
\hline $2^{\circ} \mathrm{Grau}$ & $9(45 \%)$ & $12(60 \%)$ & $14(70 \%)$ & \\
\hline 3o Grau & $5(25 \%)$ & $2(10 \%)$ & $1(5 \%)$ & \\
\hline \multicolumn{5}{|l|}{$\begin{array}{c}\text { Proteçáo } \\
\text { solar }\end{array}$} \\
\hline Chapéu & $4(20 \%)$ & $3(15 \%)$ & $5(25 \%)$ & \multirow{3}{*}{0,58} \\
\hline $\begin{array}{l}\text { Óculos } \\
\text { solares }\end{array}$ & $1(5 \%)$ & $4(20 \%)$ & $1(5 \%)$ & \\
\hline Não usa & $15(75 \%)$ & $13(65 \%)$ & $14(70 \%)$ & \\
\hline \multicolumn{5}{|l|}{ Hábitos } \\
\hline Tabagismo & $0(0 \%)$ & $0(0 \%)$ & $0(0 \%)$ & \multirow{3}{*}{0,47} \\
\hline Álcool & $4(20 \%)$ & $7(35 \%)$ & $8(40 \%)$ & \\
\hline Não usa & $16(80 \%)$ & $13(65 \%)$ & $12(60 \%)$ & \\
\hline
\end{tabular}

Referente à aplicação do questionário sobre qualidade de vida, não houve diferenças significativas entre as respostas a uma mesma pergunta, e todas foram computadas para o cálculo do escore final.

$\mathrm{Na}$ Tabela 3, ao se comparar a acuidade visual entre os grupos em funçấo do tamanho do pterígio, foi observado que no grupo I a acuidade visual em Logaritmo do Anglo Mínimo de Resolução (LogMAR) foi de $0,015( \pm 0,067)$; no grupo II, a acuidade foi de $0,080( \pm 0,132)$ e no grupo III foi de $0,067( \pm 0,127)$, com $\mathrm{p}=0,12$.

Tabela 3: Relação da acuidade visual(corrigida) entre os grupos, estratificado pelo grau de pterígio.

\begin{tabular}{ccccc} 
& Grupo I & Grupo II & Grupo III & p \\
\hline AV, & 0,015 & 0,080 & 0,067 & 0,12 \\
LogMAR & $( \pm: 0,067)$ & $( \pm: 0,132)$ & $( \pm: 0,127)$ & \\
\hline
\end{tabular}

Os dados apresentados nesta tabela são as médias dos valores encontrados, de acordo com o grupo em que cada paciente foi classificado. \pm : Desvio Padrão. Os dados foram comparados entre os grupos de através do teste de Kruskall-Wallis.

Em se tratando de escores, foi encontrado valores de $80,8( \pm 11,3)$ para o grupo I, 71,0 $( \pm 15,3)$ para o grupo II e $76,8( \pm 12,4)$ para o grupo III, com $\mathrm{p}=0,10$ (Tabela 4).
Tabela 4: Relação dos escores de qualidade visual entre os grupos, estratificado pelo grau de pterígio.

\begin{tabular}{ccccc} 
& Grupo I & Grupo II & Grupo III & p \\
Escore & $\begin{array}{c}80,8 \\
( \pm: 11,3)\end{array}$ & $\begin{array}{c}71,0 \\
( \pm: 15,3)\end{array}$ & $\begin{array}{c}76,8 \\
( \pm: 12,4)\end{array}$ & 0,10 \\
\hline
\end{tabular}

Os dados apresentados nesta tabela são as médias dos valores encontrados, de acordo com o grupo em que cada paciente foi classificado. \pm : Desvio Padrão. Utilizado o teste de Kruskall- Wallis.

Comparando-se casos unilaterais $(\mathrm{n}=29)$ versus bilaterais $(\mathrm{n}=31)$, independente do grau do pterígio, foram observados escores médios de 76,1 $( \pm 13,3)$ e 76,4 $( \pm 13,9)$, com $\mathrm{p}=0,93^{26}$.

Duas questóes do questionário de qualidade de vida NEI VFQ-25 foram avaliadas separadamente, escolhidas pela pertinência do assunto abordado referente à patologia estudada. A questão 2 questionava a percepção do paciente referente à sua visão: "Como você acha que está a sua visão? ”, e a questão 4 analisava os quesitos de dor ou desconforto nos olhos: "Você sente dor ou desconforto nos olhos?".

$\mathrm{Na}$ pergunta avaliada pela questão 2, do grupo I, $14(70 \%)$ pacientes avaliaram a visão como excelente ou boa, e somente $4(20 \%)$ pacientes do grupo II e 6 (30\%) pacientes do grupo III avaliaram dessa forma $(\mathrm{p}=0,016)$ (Tabela 5).

Tabela 5: Distribuição dos grupos em relação à percepção dos pacientes sobre sua visão.

\begin{tabular}{ccccc} 
Resposta & \multicolumn{3}{c}{ Pterígio } & Total \\
& Grupo I & Grupo II & Grupo III & \\
$\begin{array}{c}\text { Excelente ou } \\
\text { Boa }\end{array}$ & $14(70 \%)$ & $4(20 \%)$ & $6(30 \%)$ & 24 \\
Regular & $4(20 \%)$ & $9(45 \%)$ & $10(50 \%)$ & 23 \\
\hline $\begin{array}{c}\text { Ruim ou } \\
\text { Muito Ruim }\end{array}$ & $2(10 \%)$ & $7(35 \%)$ & $4(20 \%)$ & 13 \\
\hline Total & $20(100 \%)$ & $20(100 \%)$ & $20(100 \%)$ & 60 \\
\hline
\end{tabular}

Para esta tabela foi utilizado o teste exato de Fisher. $(\mathrm{p}=0,016)$

$\mathrm{Na}$ questão 4, foi observado que não houve diferença estatística entre os grupos $(\mathrm{p}=0,063)$. No grupo I, 9 (45\%) pacientes referiam desconforto moderado, assim como $10(50 \%)$ e $15(75 \%)$ pacientes, respectivamente dos grupos II e III (Tabela 6). 
Tabela 6: Distribuição dos grupos em relação à percepção dos pacientes sobre dor ou desconforto em seus olhos.

\begin{tabular}{|ccccc|}
\hline Resposta & \multicolumn{3}{c}{ Pterígio } & Total \\
& Grupo I & Grupo II & Grupo III & \\
\hline $\begin{array}{c}\text { Não sinto } \\
\text { ou Fraca }\end{array}$ & $7(35 \%)$ & $2(10 \%)$ & $1(5 \%)$ & 10 \\
\hline $\begin{array}{c}\text { Moderada } \\
\text { Severa ou } \\
\text { Muito } \\
\text { severa }\end{array}$ & $4(20 \%)$ & $8(40 \%)$ & $4(20 \%)$ & 16 \\
\hline $\begin{array}{c}\text { Total } \\
\text { Tof }\end{array}$ & $20(100 \%)$ & $20(100 \%)$ & $20(100 \%)$ & 60 \\
\hline
\end{tabular}

Para esta tabela foi utilizado o teste exato de Fisher. $(\mathrm{p}=0,063)$

\section{Discussão}

Neste estudo, observou-se que a média de idade foi acima de 40 anos para o grupo II e III. Já no grupo I foi encontrado uma média de 36,1 anos. Este dado é estatisticamente significante entre o grupo I e III, pois indica que pterígios menores poderiam estar associados a pacientes mais jovens. Em estudos de Schellini e colaboradores $^{13}$, dos 785 portadores de pterígio analisados verificou-se que a maioria encontrava-se acima de 40 anos, fato que foi comprovado também neste estudo, apesar da diferença no número de participantes abordados em cada pesquisa. Schellini relata também que $55,28 \%$ dos pacientes eram do sexo feminino. Nesta pesquisa, encontrou-se maior prevalência do sexo feminino nos 3 grupos estudados, com 13 (65\%) mulheres no grupo I e 11 (55\%) mulheres nos grupos II e III.

Alguns estudos sugerem que portadores de pele e cabelo mais pigmentados estão mais propensos a desenvolver o pterígio $^{14}$. Uma avaliação realizada em Barbados mostrou incidência superior de pterígio em negros $\left(23,4 \%\right.$ em negros e $10,2 \%$ em brancos) ${ }^{15}$. Neste estudo, nos grupos I e II a maioria era negra ou parda. O grupo III foi composto de $50 \%$ brancos e $50 \%$ negros. Não houve diferença estatística entre as frequências de raça entre os grupos estudados, talvez devido ao baixo número de participantes (n) nesta amostragem. Segundo dados do IBGE de 2010, no Tocantins, 25\% da população era da raça branca, 9,1\% negros, $63,1 \%$ pardos e 2,8\% indígenas ou amarelos ${ }^{16}$.

Em um estudo australiano ${ }^{17}$, evidenciou-se que havia maior número de portadores de pterígio em habitantes da zona rural. Teoricamente, os habitantes da zona rural teriam maior exposição à radiação solar quando comparados aos da zona urbana. Este estudo mostrou que a grande maioria residia na zona urbana ( 49 pacientes). A possível explicação para tal fato é que o local de atendimento desses pacientes é um centro de referência na capital (Palmas - TO), cuja economia principal baseia-se no setor de mão de obra para o serviço público ou privado, sendo essa a principal fonte de renda para a população. Por ser o centro administrativo e econômico do Tocantins, e devido a isso, o setor de serviços é o principal propulsor da economia palmense. A participação da agropecuária na economia palmense é menor do que a do setor de serviços ${ }^{18}$.

Em relação aos hábitos de vida, a grande maioria dos pacientes não utilizava drogas como o tabaco ou álcool. Não foi encontrado na literatura dados que poderiam se associar com os achados neste estudo. Ao ser avaliado o grau de instrução da população estudada, a maioria informou ter estudado até o segundo grau (35 pacientes). $\mathrm{O}$ índice de analfabetismo foi baixo. Esses dados podem demonstrar que a população estudada possuía bom entendimento dos questionários aplicados, fato que colaborou para validar os escores das respostas obtidas.

Quanto à média dos escores de qualidade de vida em cada grupo em função do tamanho do pterígio, encontrou-se média semelhante entre eles, que foi de 80,8 $( \pm 11,3)$ para o grupo I; $71,0( \pm 15,3)$ para o grupo II e $76,8( \pm 12,4)$ para o grupo III. De acordo com o teste estatístico aplicado, não houve diferença significante entre os mesmos $(\mathrm{p}=0,10)$, mas pelos dados percebeu-se uma discreta piora na qualidade de vida nos grupos II e III; porém, é importante ressaltar que o baixo número de participantes pode colaborar para não haver diferença estatística, uma vez que estudos que envolvem qualidade de vida são limitados. Portanto, devido ao contingente de abordagens (biopsicossociais) pode ser difícil detectar determinados padrôes estatísticos.

Entretanto, pode-se avaliar que a qualidade de vida foi alterada nos três grupos, independente do tamanho do pterígio, fato que pode contribuir para as decisóes cirúrgicas mais precoces.

A remoção cirúrgica é a única conduta disponível até o momento para a completa resolução do pterígio, e é indicada nos casos em que há prejuízo na acuidade visual, restrição da motilidade ocular, inflamaçóes crônicas, sintomas irritativos persistentes ou alteraçóes cosméticas ${ }^{19}$.

Um questionamento que se poderia fazer seria qual é o limite numérico de escores de QV que se estabeleceria como sendo bom, mediano ou ruim. Não foi encontrado padrão numérico de valores em termos de escores a ser seguido na literatura estudada.

Quanto à acuidade visual (LogMar) em relação aos 3 grupos, também não foi encontrado diferenças significativas $(\mathrm{p}=0,12)$, sendo as médias de $0,015( \pm 0,067)$ para o grupo I; média de $0,08( \pm 0,132)$ para o grupo 
II; e $0,067( \pm 0,127)$ para o grupo III. Apesar da variável acuidade visual não ter apresentado significância estatística, a percepçáo dos pacientes a respeito de sua própria visão mostrou diferenças entre os grupos. O questionário utilizado apresenta muitas questóes que abordam situaçóes cotidianas da vida dos pacientes. Nesta pesquisa, a grande maioria das acuidades visuais obtidas mostrou-se boa, fato que nos levou a não descrever e analisar separadamente a maioria das questóes do questionário, que seriam fortemente influenciadas em situaçóes com baixa visual importante e incapacitante. Sendo assim, foram analisadas duas questóes consideradas mais pertinentes com o propósito deste estudo.

A questão 2 do questionário de QV perguntava: "Como você acha que está a sua visão (com óculos ou lentes de contato, se usuário?)". Foram reagrupadas as respostas de modo a melhorar a descrição estatística. Assim foram agrupadas as opçôes "Excelente" e "Boa"; "Ruim" e "Muito ruim", e mantida de forma isolada o item "Regular". Percebeu-se que os pacientes do grupo I possuíam melhor percepção da visão se comparados aos grupos II e III $(\mathrm{p}=0,016)$, pois demonstraram que pterígios maiores, que se aproximam do eixo pupilar, tenderiam a causar maiores distorçóes ou irregularidades, o que contribui para uma pior percepção da visão, mesmo não tendo grande variaçáo da acuidade visual medida pela tabela de Snellen, método objetivo de se medir a visão. Seria interessante a combinação da acuidade visual objetivamente medida pelo médico no momento da consulta com o grau de satisfação do paciente a respeito da forma pelo qual o mesmo avalia ou percebe sua visão.

A questão 4 do questionário de QV perguntava: "Você tem sentido dor ou desconforto nos seus olhos como coceira, queimação ou dor?”. Ao responder a esta pergunta, o paciente qualificava a dor ou desconforto como moderada, fraca, severa ou muito severa. Foram reagrupadas as respostas de modo que as opçóes "Não sinto" ou "Fraca" foram agrupadas, bem como os itens "Severa" e "Muito severa", mantendo de forma isolada o item "Moderada". Um considerável número de pacientes dos três grupos se queixava de desconforto moderado, sendo que no grupo III houve maior frequência dessa resposta se comparado aos outros grupos com $\mathrm{p}=0,063$ (Tabela 6). Talvez a pouca amostragem tenha influenciado nesta avaliação e não evidenciado essa diferença entre os grupos; porém, é sugestivo que, independente do tamanho do pterígio, o nível de desconforto ou dor pode ser relativamente maior que o esperado.

O questionário aplicado neste estudo envolve uma grande abordagem geral, facilmente aplicado principalmente em patologias que alcançam um comprometimento maior da acuidade visual e que dificultam exercer atividades cotidianas. Eventualmente, a criaçáo de outro instrumento específico validado para auxiliar nas decisões terapêuticas do pterígio poderia ser factível, com um questionário mais direcionado à patologia estudada.

\section{Conclusão}

A utilização de questionários na abordagem de uma patologia ocular externa como o pterígio pode ser uma sugestâo na determinaçấo de itens que traduzam percepçáo e sentimentos dos pacientes sobre sua qualidade visual. Esses dados subjetivos referentes à percepção do paciente no que diz respeito à sua qualidade de vida podem servir de subsídios, que quando associados aos dados objetivos podem definir uma terapêutica mais adequada. Apesar da pouca amostragem, foi observado um comprometimento da qualidade de vida desses pacientes independente do tamanho do pterígio, o que poderia levar a indicaçôes mais precoces de terapêuticas cirúrgicas.

Diante dos resultados, sugerem-se novos estudos para avaliar a influência do tamanho do pterígio na qualidade de vida/visual dos pacientes.

\section{Referências}

1. Bradley JC, Yang W, Bradley RH. The science of pterygia. Br J Ophthamol. 2010;94(7):815-820.

2. Leite AO. Incidência de neoplasia intraepitelial conjuntival em pterígio [dissertação de mestrado]. Campo Grande: Universidade Federal de Mato Grosso do Sul; 2011.

3. Hosni FA. Pterygium in Qatar. Ophthalmologica. 1977;174(2):81-87.

4. Singh MM, Murthy GV, Venkatraman R, Rao SP, Nayar S. A study ocular morbidity among elderly population in a rural area of central India. Indian J Ophthalmol. 1997;45(1):61-65.

5. Taylor HR. The prevalence of corneal disease and cataracts in Australian aborigines in Northwestern Australia. Aust J Ophthalmol. 1980;8(4):289-301.

6. Ribeiro LAM, Ribeiro LFGM, Castro PRA, Silva FDL, Ribeiro VMWAM, Portes AJF, et al. Characteristics and prevalence of pterygium in small communities along the Solimóes and Japurá rivers of the Brazilian Amazon Rainforest. Rev Bras Oftalm. 2011;70(6):358-362.

7. Alves MR, Vitor G. O tratamento do pterígio. Rev Bras Oftalmol. 2005;64(5):351-362.

8. Mangione CM, Phillips RS, Lawrence MG, Seddon JM, Oray EJ, Goldman L. Improved visual function and attenuation of declines in health-related quality of life after cataract extraction. Arq of ophthalmol. 1994;112(11):14-19.

9. Kuyken W, Orley J, Hudelson P, Sartorius N. Quality of life assessment across cultures. Int J Mental Health. 1994;23(2):5-27. 
10. Ferraz EVA, Lima CA, Cella W, Arieta CEL. Adaptação de questionário de avaliação da qualidade de vida para aplicação em portadores de catarata. Arq Bras Oftalmol. 2002;65(3):293-298.

11. Simão LM, Lana-Peixoto MA, Araújo CR, Moreira MA, Teixeira AL. The Brazilian version of the 25-item national eye institute visual function questionnaire: translation, reliability and validity. Arq Bras Oftalmol. 2008;71(4):540-546.

12. Schellini SA, Shiratori CN, Spirandelli PH, Shiratori CA, Padovani CR. Intra-operative use of 5-fluorouracil in pterygium surgery. Arq Bras Oftalmol. 2000;63(2):111-114.

13. Schellini SA, Veloso CEDR, Lopes W, Padovani CR, Padovani CRP. Characteristics ospatientes with pterygium in the Botucatu region. Arq Bras Oftalmol. 2005;68(3):291-294.
14. Panchapakesan K, Hourihan F, Mitchell P. Prevalence of pterygium and pinguecula: the blue mountains eye study. Aust N Z J Ophtalmol. 1998;26(1):2-5.

15. Luthra R, Nemesure BB, Wu SY, Xie SH, Leske MC. Frequency and risk factors for pterygium in the Barbados eye study. Arch Ophthalmol. 2001;119(12):1827-32.

16. Instituto Brasileiro de Geografia e Estatística. População e condição no domicílio [acesso em 2013 Out 14]. 2010. Disponível em: ftp://ftp.ibge.gov.br/Censos/Censo_Demografico_2010/ Resultados_do_Universo/tabelas_pdf/tab1.pdf

17. McCarty CA, Fu CL, Taylor HR. Epidemiology of pterygium in Victoria, Australia. $\mathrm{Br} \mathrm{J}$ Ophthalmol. 2000;84(3):289-92.

18. Wikipédia [homepage na Internet]. Palmas [acesso em 2016 Fev 4]. Disponível em: http://pt.wikipedia.org/wiki/Palmas

19. Stern GA, Lin A. Effect of pterygium excision on induced corneal topographic abnormalities. Córnea. 1998;17(1):23-7.

\section{Como citar este artigo:}

Viegas MTC, Pinheiro SMB, Viegas FRV, Santos AFS, Diniz WG, Maia NCF. Avaliação da qualidade de vida em portadores de pterígio ocular no município de Palmas. Rev. Aten. Saúde. 2016;14(47): 5-11. 